\title{
Enhancing NMR of non-relaxing species using a controlled flow motion and a miniaturized circuit
}

\author{
Guillaume Carret, Thomas Berthelot, and Patrick Berthault* \\ NIMBE, CEA, CNRS, Université Paris-Saclay, CEA Saclay 91191 Gif-sur-Yvette, France \\ E-mail: patrick.berthault@cea.fr \\ Phone: +33 169084245
}

\begin{abstract}
In this Article we show that circulation of the sample in a closed-loop circuit combined to micro sized detection can lead to a significant signal NMR enhancement. We present an optimized NMR device based on a mini bubble-pump associated to fluidics and microdetection that can be installed on a commercial NMR spectrometer. In addition to a significant signal enhancement for slowly relaxing nuclei, we show that it enables more precise and frequent monitoring of chemical reactions. An additional modification leads to a stopped-flow system very efficient for instance for $2 \mathrm{D}$ NMR experiments with long mixing times.
\end{abstract}

\section{Introduction}

The precision and the local character of the NMR analysis arise from the low nuclear relaxation rates. While the transverse relaxation (characteristic time $\mathrm{T}_{2}$ ) needs to stay long as it really represents the signal lifetime, a slow longitudinal relaxation (characteristic time 
$\mathrm{T}_{1}$ ) is sometimes an obstacle. Numerous ways to modify the apparent relaxation time have been proposed in the literature. The first and most widespread one is the use of indirect detection via $2 \mathrm{D}$ correlation experiments in which the proton is firstly excited and then gives its polarization to the nucleus of interest. Detection can also be on the proton channel after a reverse polarization transfer, giving rise to the reverse $2 \mathrm{D}$ experiments well known for structure determination of proteins. ${ }^{1}$ Indirect detection has been successfully applied to slowly relaxing nuclei, but 1 / it may give rise to NMR sequences of long duration, 2 / it requires the presence of a magnetic coupling between these nuclei and some protons. ${ }^{2}$

The reversible addition of substances can also help in quickly initializing spin systems into their ground state. This has been used for instance in brute-force hyperpolarization of xenon-129, where paramagnetic oxygen ${ }^{33}$ or even diamagnetic liquid ${ }^{3} \mathrm{He}^{4}$ have been added to shorten the time required for xenon polarization to reach its equilibrium.

Here we show that a simple circulation of the sample in a closed-loop with controlled flow rate can increase the sensitivity per time unit. In this purpose, our recently published 3 D-printed system combining fluidics and micro-detection ${ }^{[5}$ has been used with some improvements. In this setup, a gas flow driven by a programmable syringe pump actuates a micro bubble-pump which leads to circulation of the liquid sample (Figure 1). A part of this circuit crosses the NMR detection region consisting of a solenoid.

The idea of using a flow of the solution to enhance the sensitivity of slowly-relaxing nuclei by avoiding the need to insert an interscan delay equal to several times the $T_{1}$ value is not recent (see refs 6 9), and even sophisticated recycled-flow devices associated to Dynamic Nuclear Polarization ${ }^{10}$ have been proposed. But these setups were always associated to large sample volumes. Here the full solution volume is on the order of 250 microliters (it can be further reduced to $100 \mu \mathrm{L}$ ), and while the detected volume is in the range $2.5-16 \mu \mathrm{L}$ (see the Experimental Section), the liquid flow in a closed-loop circuit makes that all the solution is useful. This setup compares favorably with others in terms of mass sensitivity (i. e. for mass-limited samples). 


\section{Results and discussion}

As the principle of the mini bubble-pump is already described in ref. 5, we will only recall here its basic principle and the evolutions that have been made to improve it. A schematic drawing of its operation mode and a 3D rendering of the last design are given in Figure 1. Gas bubbles exiting from the inlet pipe create a flow motion for the liquid (blue arrows). More details are given in the Experimental Section. Mainly two prototypes differing by the diameter of the fluidic channels and measurement capillary (designs $A$ and $B$ ) have been built.

Our first task has been to characterize the sample solution flow rate. In this purpose, ${ }^{1} \mathrm{H}$ NMR velocimetry experiments have been performed on water with our setup (Fig. 2). They show that the liquid flow rate is correlated to the gas flow rate. The curve displaying the solution flow rate as a function of the gas flow rate has an exponential behavior, a plateau occurring for gas flow rates exceeding $1.6 \mathrm{~mL} / \mathrm{min}$ for design $A(1 \mathrm{~mL} / \mathrm{min}$ for design $B)$. Using gas flow rates higher than this value is thus useless, whatever the gas used. For design $A$, the asymptotic value corresponds to a liquid speed of $27 \mathrm{~mm} / \mathrm{s}(24 \mathrm{~mm} / \mathrm{s}$ for design $B)$.

As an example of the performance of the approach for slowly relaxing nuclei, a first experiment is performed with a $200 \mathrm{mM}{ }^{13} \mathrm{C}$-enriched urea sample in $\mathrm{D}_{2} \mathrm{O} .250 \mu \mathrm{L}$ of this solution are inserted in the NMR cell equipped with a 8-turn solenoid tuned at $125.76 \mathrm{MHz}$ $\left({ }^{13} \mathrm{C}\right.$ Larmor frequency at 11.74 Tesla). The fluidic system is installed. Figure 3 a displays the ${ }^{13} \mathrm{C}$ spectrum acquired in 16 scans preceded by 2 dummy scans. The inter-scan repetition time is $2 \mathrm{~s}, 90^{\circ}$ flip angle pulses are used, and this spectrum is recorded in the absence of gas flow. Due to slow relaxation of the carbonyl carbon $\left({ }^{13} \mathrm{C} \mathrm{T}_{1}\right.$ on the order of $\left.39 \mathrm{~s}\right), 11$ as expected no signal appears on the spectrum. Figure $3 \mathrm{~b}$ displays the ${ }^{13} \mathrm{C}$ spectrum acquired in exactly the same experimental conditions, except that it is recorded in the presence of an air flow of $1 \mathrm{~mL} / \mathrm{min}$ (leading to a liquid flow rate $k_{l}$ estimated at ca. $2 \mathrm{~cm} / \mathrm{s}$ according to Fig. 2). The carbonyl signal now appears with a good signal-to-noise ratio (SNR $=21$ when Lorentzian apodization of $10 \mathrm{~Hz}$ is applied before Fourier transformation). With some more 
signal accumulation, we have checked that no significant line broadening occurs due to the flow, ${ }^{12}$ as expected as the liquid circulation speed is not too fast. Indeed, considering :

$$
\frac{1}{\pi T_{2}^{\text {flow }}}=\frac{1}{\pi T_{2}^{\text {static }}}+\frac{k_{l}}{l_{c}}
$$

$\left(k_{l}\right.$ : liquid flow rate, estimated from the calibration experiments of Figure $2 l_{c}$ : detection length, equal to $0.5 \mathrm{~cm}$ for the ${ }^{13} \mathrm{C}$ coil), for a value of $\frac{1}{\pi T_{2}^{\text {static }}}$ of $85 \mathrm{~Hz}$ it leads to a line broadening of less than $4 \%\left(\frac{k_{l}}{l_{c}}=20 / 5=4 \mathrm{~Hz}\right)$.

Note that whereas for small molecules $T_{1}$ is equal to $T_{2}$ (the present case), for larger molecules where $T_{1}>T_{2}$, an improvement in signal-to-noise ratio might be realized even with fast circulation of the solution without any line broadening effect.

In any case, in our cell, the volume of the sample solution $\left(V_{\text {sol }}\right)$ is $250 \mu \mathrm{L}$. In order to totally refresh the solution in the NMR detection area (of volume $V_{\mathrm{NMR}}$ ) between two successive scans (separated by a delay $T$ ), a minimal liquid flow rate $k_{l}$ equal to $V_{\mathrm{NMR}} / T$ is required. With the current micro-coil with inner volume of $2.5 \mu \mathrm{L}$ and a repetition time of 2 seconds, it corresponds to $k_{l}=0.075 \mathrm{~mL} / \mathrm{min}=0.25 \mathrm{~cm} / \mathrm{s}$. This is easily achieved with our set-up with a volumic gas flow rate value of $0.1 \mathrm{~mL} / \mathrm{min}$ according to the ${ }^{1} \mathrm{H}$ NMR velocimetry experiments. Action of the syringe pump during 200 seconds - in continuous or stopped-flow mode - is necessary in order the full solution to cross the NMR area.

Intuitively it could be thought that separating the sample in 100 parts individually detected in 100 successive acquisitions separated by an interscan delay $T$ is equivalent to detecting the whole sample at one time and waiting 100 times $T$, provided that $100 \times T$ is still lower than $T_{1}$. But in the first approach, several effects can combine favorably. Firstly the filling factor is optimized, the detection coils being as close as possible to the sample. Secondly, during the loop different effects are susceptible to increase the apparent liquid relaxation rate.

In order to assess the sensitivity gain afforded by our setup, we have compared the 
SNR obtained with our setup with the one obtained with a standard $8 \mathrm{~mm}$ NMR tube in the original commercial micro-imaging probehead equipped with a ${ }^{13} \mathrm{C}$ saddle coil (optimal filling factor) , using the same ${ }^{13} \mathrm{C}$-enriched urea concentration. For that, FIDs have been recorded in a $32 \mathrm{~s}$ time window with different inter-scan delays and flip angle pulses. For the ${ }^{13} \mathrm{C}$ signal of the urea carbonyl group, in the case of scans separated by 2 seconds, the optimal ${ }^{13} \mathrm{C}$ flip angle, also known as the Ernst angle ${ }^{13}$ and whose expression for a static solution is $\alpha_{E}=\arccos \left(\exp \frac{-T_{R}}{T_{1}}\right)$, is equal to $18.2^{\circ}$. For a gas flow of $1 \mathrm{~mL} \cdot \mathrm{min}^{-1}$, in looking for a maximum signal, we have found an optimal flip angle of $60^{\circ}$, leading to an apparent relaxation time of $3 s$. In Fig. S4 of the Supp. Info. we have then compared the SNR obtained with the flowing solution both with the static case and the classical $8 \mathrm{~mm}$ tube, at their respective optimal excitation angle. The given values are given after correction by the total sample volumes and can therefore be denoted as mass-corrected SNR. By comparing the SNR obtained in the static case, both with our system and the $8 \mathrm{~mm}$ tube, we find that the SNR obtained with the NMR tube is 4.3 times higher, with an amount of solution being also 4 times higher. This means that for a given total volume of solution the SNR is similar, even though the detected volume in our system is only $2.5 \mu \mathrm{L}$, thanks to our optimized coil. Moreover, we can observe a further 3 fold increase in SNR with our system when the solution is flowing, compared to the static case. When taking this into account, we can conclude that our flowing system increases the SNR for a given quantity of sample by a factor of 3 , compared to a commercial probe. Considering that the detected volume is only $2.5 \mu \mathrm{L}$, the sensitivity improvement due to our system could be further improved either by reducing the overall volume, or by increasing the detected volume. Finally, for the solution flowing at $1 \mathrm{~mL} \cdot \mathrm{min}^{-1}$ the fact that the flow is laminar makes that the liquid near the capillary walls has no significant displacement (the liquid speeds plotted in Fig. 2 are average values) and therefore the optimal excitation angle is not $90^{\circ}$.

We have been unable to find evidence of a 'memory effect' for the flowing solution, i.e. even after a complete loop the apparent relaxation seems the same (no saturation of the 
magnetization is observed). Several carrier gases have been tested: air, pure $\mathrm{N}_{2}$, pure $\mathrm{O}_{2}$, xenon. Their nature (density, water solubility) does not seem to act significantly on the signal-to-noise ratio, thus this is neither a question of paramagnetic relaxation enhancement nor an effect linked to large magnetic susceptibility variation induced by the air liquid interface. Also, interaction with the polymer of the poly(methyl methacrylate) type incompletely reticulated or releasing paramagnetic species could be taken as responsible of the longitudinal relaxation shortening. But this possibility has also been discarded after some NMR experiments on polymer pieces. Moreover our last prototypes in which the walls of the liquid circuit have undergone a special treatment preventing relaxation show the same behavior. Another explanation could reside in the bursting of gas bubbles that has been shown by Takahashi et al. ${ }^{14}$ to generate free-radicals even in the absence of a harsh dynamic stimulus. But some experiments performed with biological cell suspensions did not reveal any increased cell mortality. Finally it is likely that a strong mixing of the solution occurs in the upper part of the circuit, where the bubbles quit the solution. Thus here for the studied nuclei and compounds, the use of immobilized free radicals $\frac{12115}{177}$ was not required, although these relaxation agents could have been easily placed in the upper part of the circuit.

Given the easiness to plug different 3D-printed inserts equipped with solenoids having their own resonance frequency on the micro-imaging probe base, other experiments exemplifying the gain afforded by this approach have been performed in ${ }^{7} \mathrm{Li} \mathrm{NMR}$. For the ${ }^{7} \mathrm{Li}$ NMR spectra of $\mathrm{LiCl}$ also a significant gain in signal-to-noise ratio is also observed between the static and the on-flow experiments (see Fig. S5 of the Supp. Info.).

The approach of using a circular flow has also a great interest for the continuous monitoring of chemical reactions, $\frac{18}{18}$ and particularly reaction involving modification of quaternary carbons. A classical way to follow such reactions inside the NMR spectrometer would be to acquire ${ }^{13} \mathrm{C}$ NMR spectra at regular time intervals. In this domain the use of hyperpolarized species can be helpful and fast ${ }^{13} \mathrm{C}$ NMR acquisitions separated by randomized pulsed field gradients in order to remove residual coherences from the preceding scans can be used.19 
But circulation of the sample in a closed-loop circuit can be a good alternative. The flow motion homogenizes the reactant mixture, and by the same principle as previously described detection of poorly relaxing nuclei is facilitated. To demonstrate this, we have chosen the esterification of isopropanol by acetic anhydride in the presence of $\mathrm{H}_{2} \mathrm{SO}_{4}$. This chemical reaction has been monitored via ${ }^{13} \mathrm{C}$ NMR in the same conditions in a classical dual ${ }^{13} \mathrm{C}-{ }^{1} \mathrm{H}$ probe head (5 mm o.d. NMR tube) and in our flow cell equipped with the same solenoid as previously. Figure 4 displays the time evolution of the product and reactant low field ${ }^{13} \mathrm{C}$ signals (carbonyls). A gain in sensitivity by a factor of $c a .2 .8$ in favor of the on-flow experiment is observed, while a volume of only $16 \mu \mathrm{L}$ is detected (insert of design $B$ ) instead of $400 \mu \mathrm{L}$ for the classical static experiment. Also it is clear from Figure $4 \mathrm{~A}$ that for the static experiment the first steps of the reaction are missed (the signals of the products are not null at the beginning of the NMR experiments). Conversely it is not the case for our system (Figure 4B). Sulfuric acid is inserted in the gas inlet of the flow cell, it is mixed with the solution when the flow starts, allowing the whole reaction to be observed. Indeed the product signals here start from zero. Note that our setup would also enable installation of multiple coils along the solution circuit, which has been proved of interest for the monitoring of fast reaction kinetics. 20

Using a continuous flow circuit to enhance the NMR signal of slowly relaxing species can however be problematic for experiments where the acquisition does not occur immediately after the first pulse. In this case, at the beginning of the acquisition many spins that have been excited are outside the coil at the detection step. For instance, the sensitivity of a 2D experiment with a mixing time would crucially depend on the flow rate; this effect would become even more dramatic in the case of magnetic field gradients used for coherence selection. To overcome this problem it is important to be able to stop on purpose the flow with a precise control and a short response time. For this reason, upstream to the NMR cell we have introduced a pneumatic valve that is driven by the NMR pulse sequence (see Fig. 5). 
In order to assess the efficiency of the stopped-flow method with respect to experiments performed without flow or with a continuous flow, two-dimensional ${ }^{13} \mathrm{C}$-TOCSY experiments have been performed in the three modes on a $40 \mathrm{mM}$ aqueous solution of glucose uniformly enriched in ${ }^{13} \mathrm{C}$ at $99 \%$. Figure 6 and Figure $\mathrm{S} 6$ display the contour plots of the stopped-flow, static and continuous flow experiments taken at the same noise level. The three experiments have the same duration (see Experimental Section). Even if the static field homogeneity was not good for these experiments, in particular in the region of the anomeric carbons, we can clearly see that the stopped-flow experiment (A) is more sensitive than the static (B) and continuous flow $(\mathrm{C})$ experiments. Also a detailed assignment task in the $70-80 \mathrm{ppm}$ region is easier with the experiment $\mathrm{A}$.

Our system is intended to be low cost, and for precious solutions the insert can easily be replaced by another insert. However for replacing only the sample in our setup, changing the sample is not a problem, as it is possible to insert the cone of a pipetman into the outlet hole - thereby achieving an airtight connection - and to aspire the liquid. The washing of the NMR cell is performed by flushing many times with the solvent and drying.

\section{Conclusion}

The approach of sample circulation in a closed-loop with controlled flow rate is fully different from that of LC-NMR. Here, low amounts (100 to $250 \mu \mathrm{L}$ ) of solution are required in the basic version of the micro-pump. With respect to the capillary NMR (CapNMR) technique, 21 there are two major differences: i) the solution crosses several times the detection area, ii) the use of a small amount of additional solvent designed to push the sample through the inlet line into the flow cell is not necessary. It is therefore less likely to pollute the solution of interest.

The NMR cell is 3D-printed and although it has been conceived to be plugged onto a classical Bruker micro-5 probehead body, it could be adapted to other probehead bod- 
ies. Therefore it is cheap, it can be easily replaced, and different nuclei can be studied in NMR spectroscopy or imaging, with simple modification of the tuning/matching capacitors. The NMR sensitivity, which is a concern for hyphenated NMR techniques as stated by the emergence of LC-NMR cryoprobes, is here improved by the use of solenoidal microcoils more efficient than the saddle coils of classical probeheads and presenting an excellent filling factor.

Obviously a non-magnetic peristaltic pump (with a remote motor) could have been employed to induce circulation of the sample solution, but the present solution is cheaper and more versatile, as the carrier gas can also be used to enrich the solution, as a chemical reactant, or as a nutriment for living organisms.

It presents many interesting features, in particular for slowly-relaxing nuclei for which the gain in signal-to-noise ratio per time unit can be consequent. Among different applications, monitoring of chemical and biochemical reactions (e.g. enzymatic catalysis) will find interest in this approach.

The basic version of our NMR cell can largely be improved. First, the spectral resolution has to be increased (even if in the current approach no bubble is present in the detection area, see ref. 5). The envisioned solutions for that are to equip the insert with a support ensuring perfect positioning of the micro-coil, and to surround the micro-coil with a fluid having magnetic susceptibility close to that of the solvent. ${ }^{222}$ Given the current geometry of our system, this will be performed without too much difficulty. Also, in our current set-up, even in continuous flow mode the solution progresses with jerks, as the 'motor' are the gas bubbles. Even if in continuous mode the trouble is minor, it could become problematic for some on-flow studies (see for instance the noisy character of the data points in Fig. S3). In addition this can be an inconvenience for a more fundamental study aiming at understanding the influence of the nature of the carrier gas (solubility, density, etc.) on the solution speed. To counteract this jerky behavior, we will use recent solutions from the literature. 23

Today a unique solenoidal coil tuned at one frequency is used; our wish is to be able to excite and detect several nuclei. This will enable for instance to decouple proton while 
observing the other nucleus. Either a multi-tuned coil or a system with several embedded $\operatorname{coil}^{24}$ will be developed. Finally other inlets can be installed in the same insert to enable the real-time monitoring of multi-partners chemical reactions. Given the unused space available in the current version of the prototype, this does not present too much difficulty.

Such improvements are underway in our laboratory. 

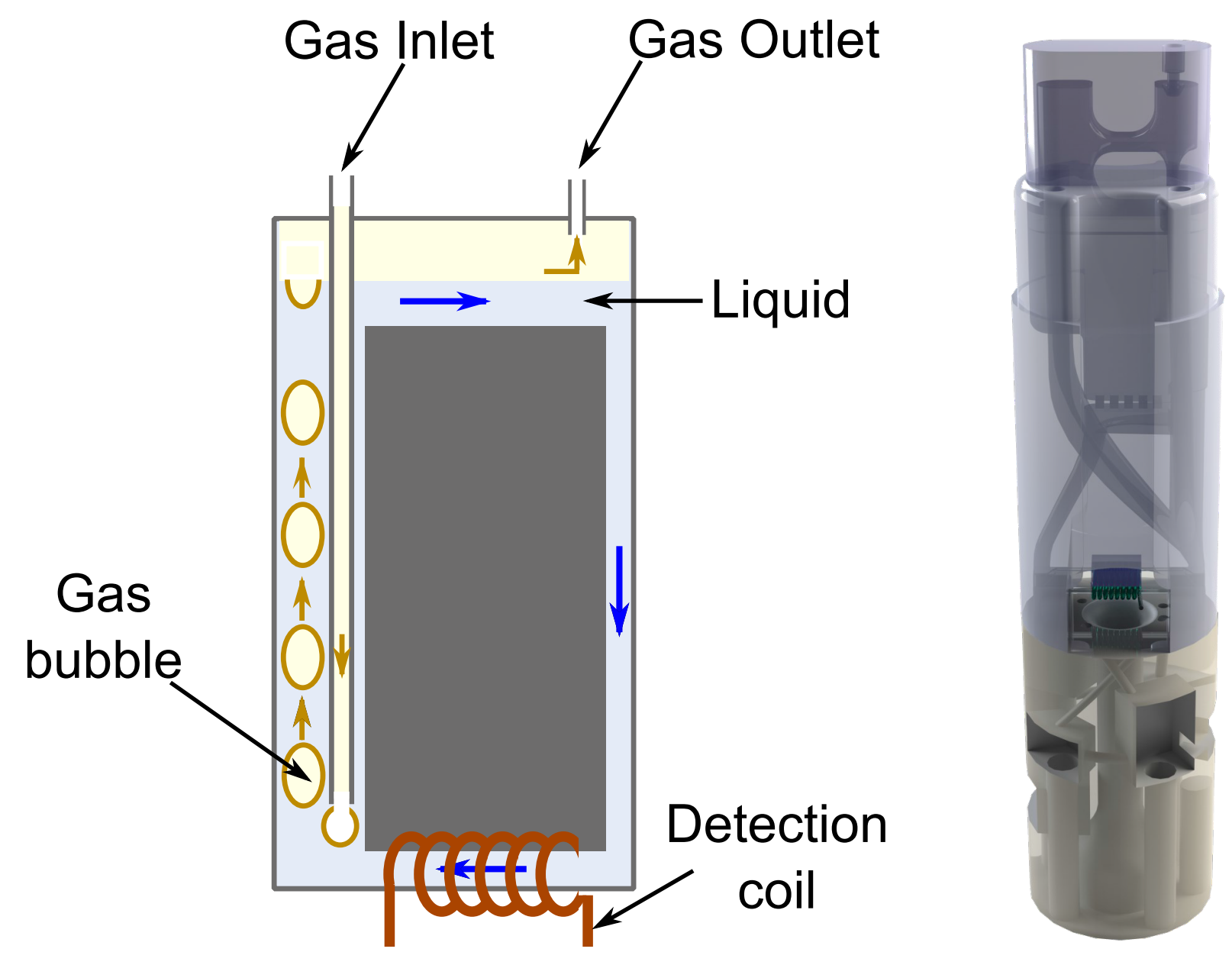

Figure 1: Left: principe of the mini bubble-pump. This 3D-printed piece is plugged onto the body of a Bruker micro-5 probehead, the solenoid is connected to its electronic circuit, and the gas inlet tube is connected to the syringe pump. Right: 3D rendering of one of our - more evolved - prototypes (diameter $18 \mathrm{~mm}$ ). Two polymers are printed in 3D; the first one of ABS-type for the capacitors and the connection to the probehead, the second one of PMMA-type for the microfluidics channels. 


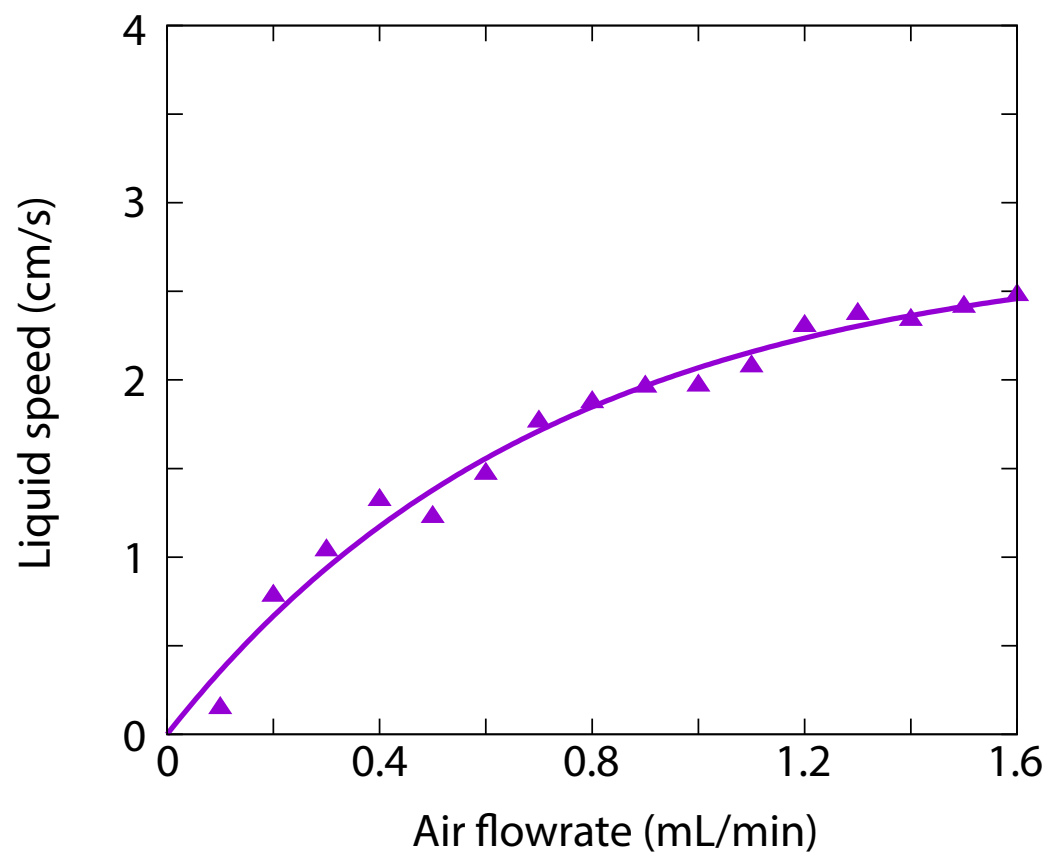

Figure 2: Liquid speed in the ${ }^{1} \mathrm{H}$ detection coil, as measured by the velocimetry experiments, as a function of the setpoint air flowrate $n$ the syringe (insert of design $A$ ). 


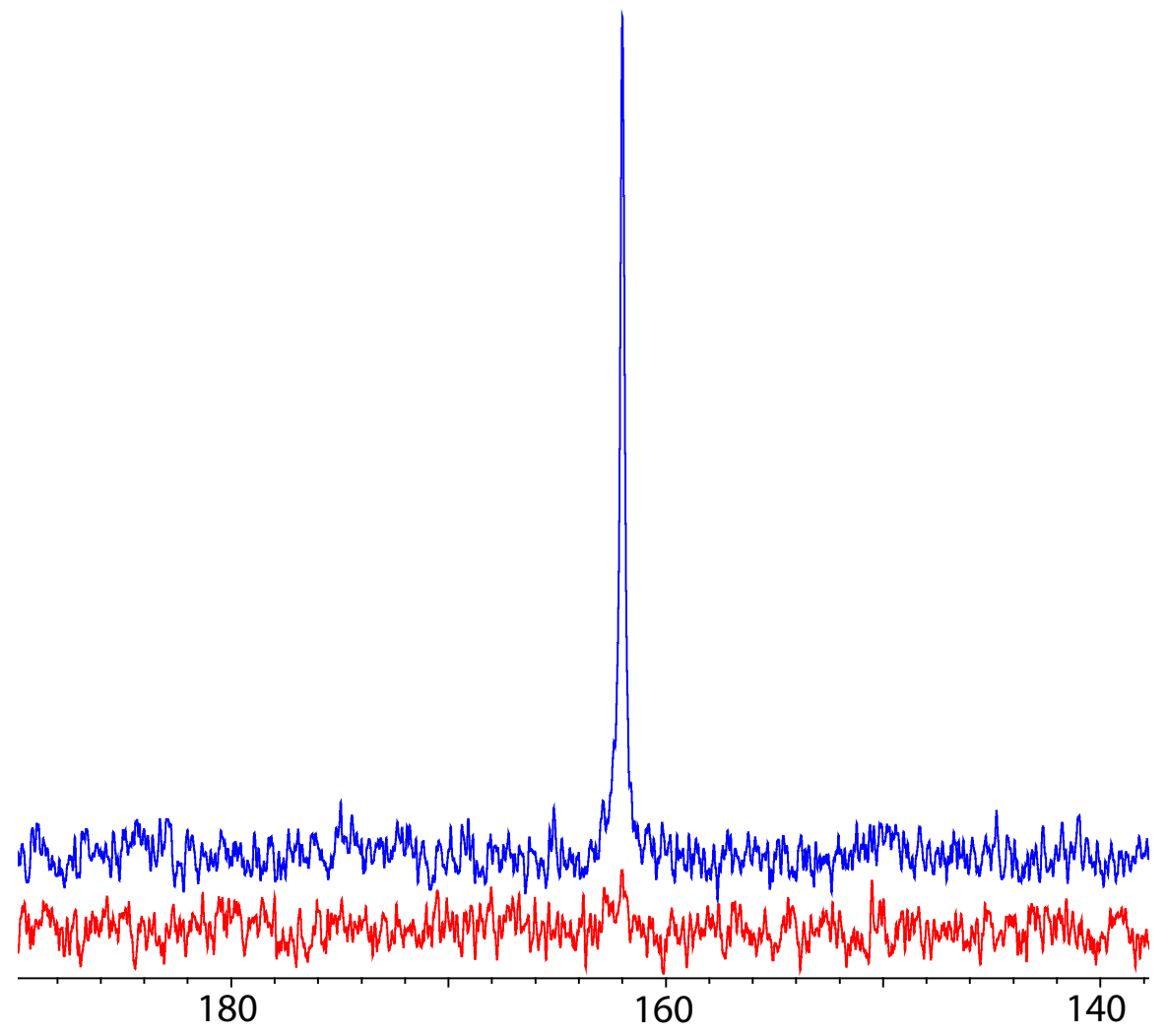

Figure 3: ${ }^{13} \mathrm{C}$ NMR spectrum of $2.5 \mu \mathrm{L}$ of a $200 \mathrm{mM}$ solution of ${ }^{13} \mathrm{C}$-enriched urea in $\mathrm{D}_{2} \mathrm{O}$, a) for a static solution, b) with flow. Both spectra have been recorded in 16 scans preceded by 2 dummy scans, with an inter-scan delay of $2 \mathrm{~s}$. 

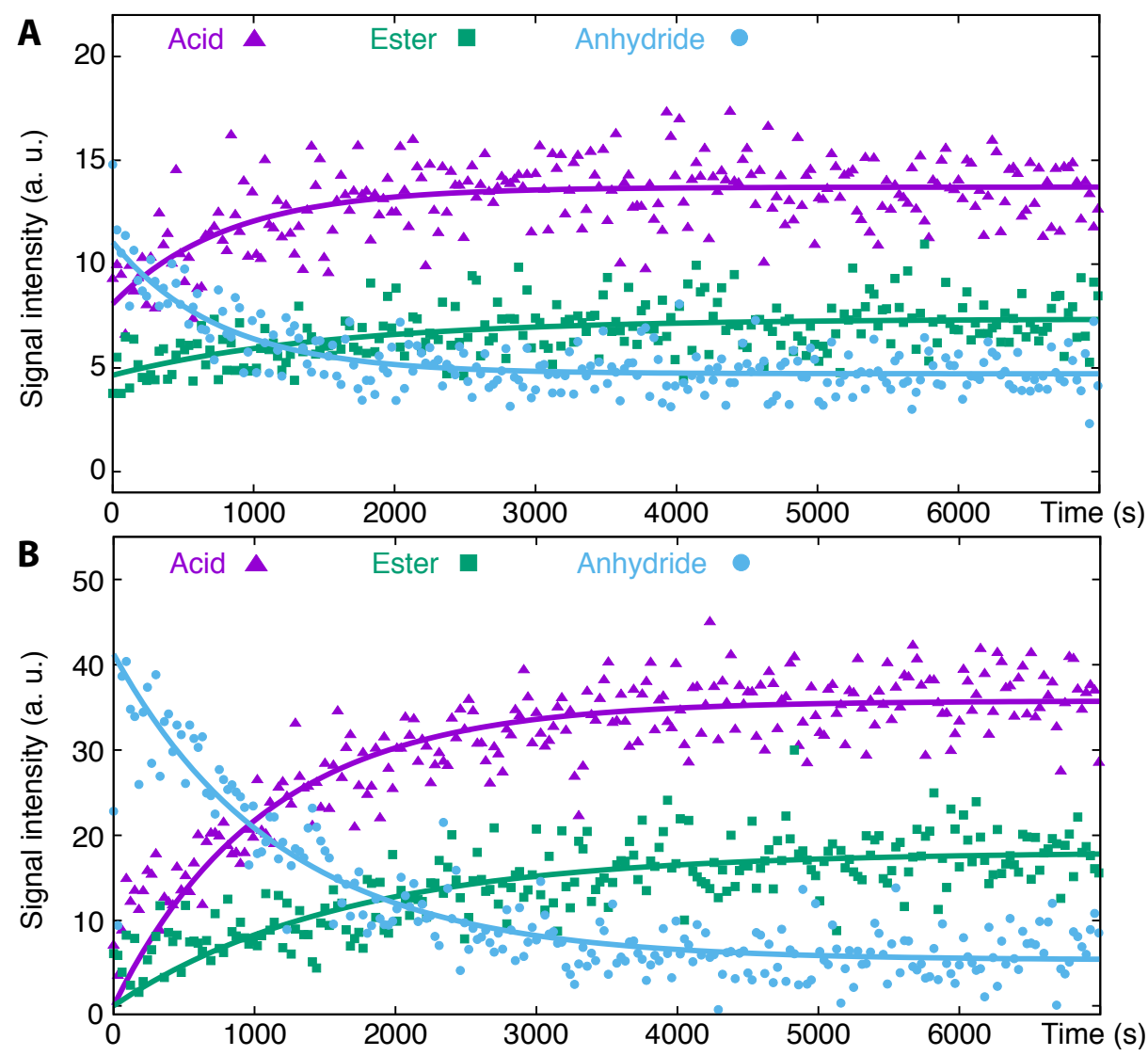

Figure 4: Time evolution of the ${ }^{13} \mathrm{C}$ signals of the reactant and the products during the esterification reaction (the trend curves are added). A) With the reaction occurring in a 5 mm o.d. NMR tube, B) with our system and a gas flow rate of $1 \mathrm{~mL} . \mathrm{min}^{-1}$. The signal intensities are given in arbitrary units, but are the same between $\mathrm{A}$ and $\mathrm{B}$ and can therefore be compared. 


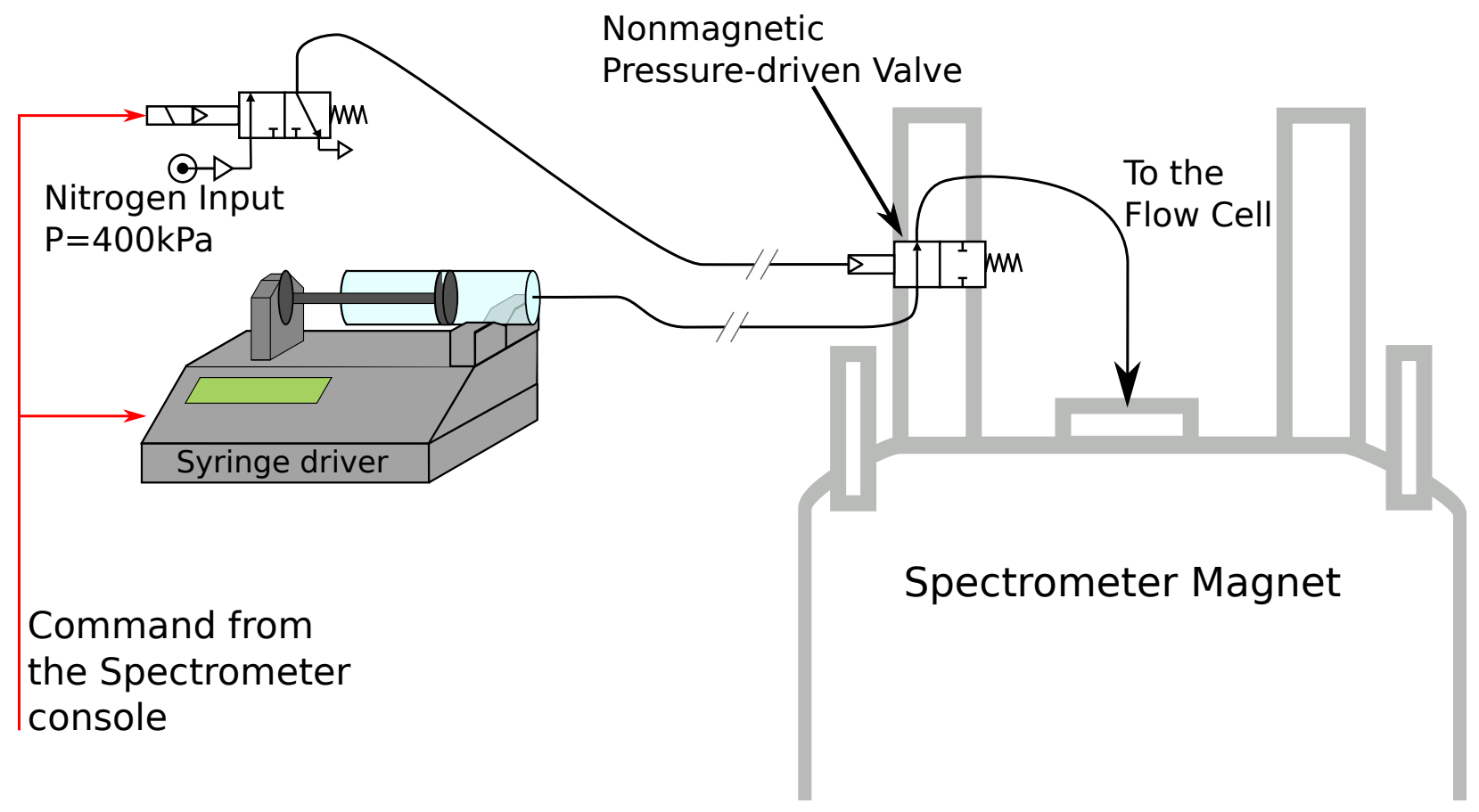

Figure 5: Scheme of the stopped flow device installed upstream to the micro-bubble pump. 

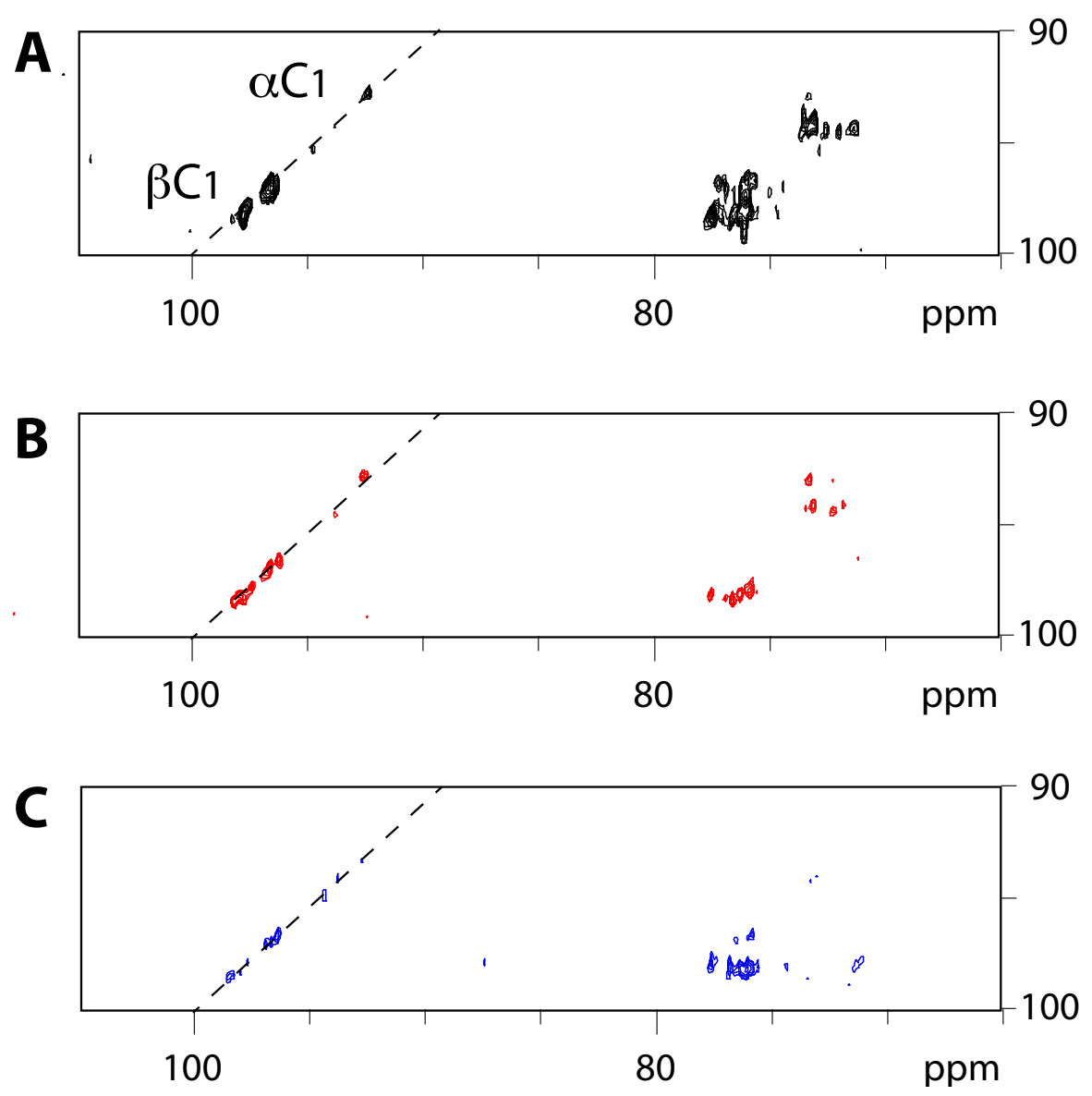

Figure 6: 2D ${ }^{13} \mathrm{C}$-TOCSY experiments without ${ }^{1} \mathrm{H}$ decoupling recorded with $\mathrm{A}$ ) the stoppedflow mode, B) no flow, and C) continuous flow, on a sample of uniformly ${ }^{13} \mathrm{C}$ enriched glucose at $40 \mathrm{mM}$ in $\mathrm{D}_{2} \mathrm{O}$. Each experiment spent 2 hours 8 minutes. Raw matrices of $2048 \times 200$ complex data points. Zoom in on the $\mathrm{C} 1-\mathrm{C} 2$ correlation region. 


\section{Experimental}

\section{Micro bubble-pump and NMR system}

A programmable amagnetic syringe driver (Harvard Apparatus PHD2000) is placed on top of the 11.7 T narrow bore NMR magnet (Bruker BZH 500/52) and is interfaced with the spectrometer console. It pushes on a $120 \mathrm{~mL}$ syringe filled with air, injecting it in the NMR cell via a PTFE tubing (Adtech Bioblock). The cell is printed with an Objet30 Pro inkjet 3D-printer (Stratasys), using a transparent resin (VeroClear). In this cell, the air is carried through a PEEK capillary (Upchurch, i.d. $0.8 \mathrm{~mm}$ ) before being released into the solution. The capillary seats in a printed channel, thus simplifying the assembling process. Two different systems were built with the detection region constituted either of a $0.8 \mathrm{~mm}$ inner diameter (design $A$ ) or of a $2 \mathrm{~mm}$ inner diameter (design $B$ ) quartz tube (CM Scientific) fitted inside an air-core solenoid (Midi Springs from Coilcraft). The length of the quartz tube covered by the rf coil is $0.5 \mathrm{~cm}$ for the ${ }^{1} \mathrm{H}$ coil, $0.5 \mathrm{~cm}$ for the ${ }^{13} \mathrm{C}$ coil. These setups enable detection of $2.5 \mu \mathrm{L}$ for design $A$ and $16 \mu \mathrm{L}$ for design $B$. For proton, rf inductor values of $22 \mathrm{nH}$ were used, while (for instance) for ${ }^{13} \mathrm{C}$ rf inductor values of $150 \mathrm{nH}$ were used. Prior to any experiment, $250 \mu \mathrm{L}$ of solution were injected in the cell and recirculated to eliminate every possible bubble in the detection region.

All the NMR experiments were performed on a narrow bore $11.7 \mathrm{~T}$ Bruker spectrometer $\left({ }^{1} \mathrm{H}\right.$ frequency: $\left.500.13 \mathrm{MHz}\right)$ at room temperature. Each 3D-printed NMR cell, with its own design and capacitance value was plugged onto the body of a Bruker micro-5 (microimaging) probe head, and the gradient sleeve then surrounded it. A GREAT 3/40 gradient system unit (delivering a maximum value of $200 \mathrm{G} / \mathrm{cm}$ for a current of $40 \mathrm{Amps}$ ) was used. The software for acquisition and processing was Bruker TopSpin 3. 


\section{Velocimetry experiments}

In order to calibrate the gradients and delays for the flow velocimetry, a reference experiment was performed. The micro-5 probe head was installed in the spectrometer with a dual ${ }^{13} \mathrm{C}-{ }^{1} \mathrm{H}$ insert. A PTFE tube of inner diameter $0.81 \mathrm{~mm}$ was inserted in a $8 \mathrm{~mm}$-diameter NMR tube (making a loop at the bottom of it). The programmable syringe pump then pushed the water doped in $\mathrm{CuSO}_{4}$ placed in the syringe into the PTFE tube at defined flow rates between 0.02 and $2 \mathrm{~mL} / \mathrm{min}$. A stimulated echo sequence with a slice selection along $z$ of $10 \mathrm{~mm}$, an inter-gradient delay of $10 \mathrm{~ms}$ and gradient pulses of $1 \mathrm{~ms}$ along the $z$ axis with amplitudes stepped from -64 to $+64 \mathrm{G} / \mathrm{cm}$ in 128 increments was used. In order to observe the flow speed distribution along the axial dimension of the PTFE tube, a $10 \mathrm{G} / \mathrm{cm}$ gradient along $x$ was applied during acquisition. The resulting matrices after double Fourier transformation and the curve giving the liquid speed as a function of the setpoint flow rate programmed to the syringe pump are displayed in Supp. Info. (Figs. S1 and S2). See also Supp. Info. for the details of the processing procedure.

For the velocimetry experiments in the NMR cell, $250 \mu \mathrm{L}$ of water doped with $\mathrm{CuSO}_{4}$ were placed in a ${ }^{1} \mathrm{H}$-tuned insert, which was then plugged onto the micro-5 probe head. The syringe was filled with air and the measurements were acquired using the same scheme as before, except that no slice selection is performed and that the velocimetry encoding and decoding gradients were applied along $y$. We had checked before this experiment that the axis of the capillary corresponded to the axis of the $y$ gradient.

\section{Slowly-relaxing nuclei}

A ${ }^{13} \mathrm{C}$-tuned insert of design $A$ (detection volume $2.5 \mu \mathrm{L}$ ) was filled with a solution of $99 \%$ ${ }^{13} \mathrm{C}$-enriched urea (from EurisoTop) at $200 \mathrm{mM}$ in $\mathrm{D}_{2} \mathrm{O}$. Single acquisitions with 16 scans and an inter-scan delay of $32 \mathrm{~s}$ were performed in the static and in the on-flow mode (setpoint gas flow value: $1 \mathrm{~mL} / \mathrm{min}$ ). 


\section{Chemical Reaction}

For the esterification reaction, the same reactant proportions were kept between the static experiment with a classical probe head and the on-flow experiment with our home-built system. $354 \mu \mathrm{L}$ of 2-propanol (Sigma-Aldrich) were mixed with $132 \mu \mathrm{L}$ acetic anhydride (Sigma-Aldrich). $250 \mu \mathrm{L}$ of this solution were put in the 3D-printed NMR cell of design $B$.

The difference in the protocol was the following. For the static experiment, in the $5 \mathrm{~mm}$ o.d. NMR tube $8 \mu \mathrm{L}$ of $\mathrm{H}_{2} \mathrm{SO}_{4} 96 \%$ (Sigma-Aldrich) were added to the reactant solution, the tube immediately put inside the NMR magnet, and the 2D NMR experiment started without further delay. The NMR insert was installed onto the micro-5 probehead body. Then $4 \mu \mathrm{L}$ of $\mathrm{H}_{2} \mathrm{SO}_{4} 96 \%$ were introduced in the gas inlet capillary. The probe head was connected to the syringe pump and installed inside the NMR magnet, and the NMR started without further delay. The NMR pulse sequence was written as a $2 \mathrm{D}$ matrix, each ${ }^{13} \mathrm{C}$ acquisition being stored on one raw (interscan delay: $2 \mathrm{~s}, 8$ scans per raw, delay of $30 \mathrm{~s}$ between two raws). The direct dimension contained $32 \mathrm{~K}$ data points, the indirect one 256 points. The syringe pump was started simultaneously with the NMR sequence (continuous infusion with a gas flow rate of $1 \mathrm{~mL} / \mathrm{min}$, see Supp. Info.).

\section{Stopped-flow system}

In order to quickly stop the gas flow, an interface was designed to control both the syringe driver and a solenoid valve using a single TTL output of the spectrometer. Since we could not directly use a solenoid valve in the vicinity of the magnet, a pressure-driven membrane valve (Takasago model PMDP-3-M6KG) was chosen in this purpose. This valve was controlled using a 3/2 solenoid valve (Festo 7802 MFH-2-1/8), as shown in Figure 5. This allowed us to stop the flow quasi-instantly, while maintaining the correct working pressure in the syringe.

For comparison of the stopped-flow mode with the continuous flow and static modes, $2 \mathrm{D}{ }^{13} \mathrm{C}$ TOCSY experiments with a short mixing time of $4.6 \mathrm{~ms}$ (only ${ }^{1} J_{C C}$ correlations) were performed on a $40 \mathrm{mM}$ solution of $\mathrm{U}^{13} \mathrm{C}$ glucose (EurisoTop) in $\mathrm{D}_{2} \mathrm{O}$. For each of the 
200 raws recorded in the indirect dimension, 32 scans were accumulated with an inter-scan delay of 1.18 s. For the stopped-flow and continuous-flow experiments, a gas flow value of 1 $\mathrm{mL} / \mathrm{min}$ was used. In the former experiment, the gas was pushed during $0.5 \mathrm{~s}$ followed by a resting delay of $0.5 \mathrm{~s}$, at the beginning of each scan. We checked afterwards that the second delay could have been shorten to $0.1 \mathrm{~s}$ without too much effect.

\section{Notes}

The authors declare no competing financial interest.

\section{Acknowledgement}

Support from the French Ministry of Research (project ANR- 12-BSV5-0003 MAX4US) is acknowledged.

\section{Supporting Information Available}

- Details and equations for the ${ }^{1} \mathrm{H}$ NMR velocimetry experiments.

- $2 \mathrm{D}{ }^{1} \mathrm{H}$ velocimetry (calibration and experiment for the insert of design $\mathrm{B}$ ).

- Comparison of the SNR obtained with a classical probehead and our setup at various flowrates.

- ${ }^{7} \mathrm{Li} \mathrm{NMR}$ spectra of $\mathrm{LiCl}$ in water recorded with and without flow.

- Comparison of ${ }^{13} \mathrm{C}$-TOCSY experiments recorded with the stopped-flow, static, and continuous flow modes.

This material is available free of charge via the Internet at http://pubs.acs.org/. 


\section{References}

(1) Ikura, M.; Kay, L. E.; Bax, A. Biochemistry 1990, 29, 4659-4667.

(2) Löble, M. W.; Casimiro, M.; Thielemann, D. T.; Oña-Burgos, P.; Fernández, I.; Roesky, P. W.; Breher, F. Chem. Eur. J. 2012, 18, 5325-5334.

(3) Honig, A.; Wei, X.; Lewis, A.; ter Haar, E.; Seraji-Bozorgzad, K. Physica B 2000, 284-288, 2049-2050.

(4) Krjukov, E. V.; O’Neill, J. D.; Owers-Bradley, J. R. J. Low Temp. Phys. 2005, 140, $397-408$.

(5) Causier, A.; Carret, G.; Boutin, C.; Berthelot, T.; Berthault, P. Lab. Chip 2015, 15, 2049-2054.

(6) Laude, D. A.; Lee, R. W.; Wilkins, C. L. J. Magn. Reson. 1984, 60, 453-459.

(7) Laude, D. A.; Lee, R. W. K.; Wilkins, C. L. Anal. Chem. 1985, 57, 1281-1286.

(8) Laude, D. A.; Lee, R. W.; Wilkins, C. L. Anal. Chem. 1985, 57, 1286-1290.

(9) Sudmeier, J. L.; Günther, U. L.; Albert, K.; Bachovchin, W. W. J. Magn. Reson. A 1996, 118, 145-156.

(10) Stevenson, S.; Glass, T.; Dorn, H. C. Anal. Chem. 1998, 70, 2623-2628.

(11) Moreland, C. G.; Carroll, F. I. J. Magn. Reson. 1976, 23, 167-169.

(12) Fischer, H. H.; Seiler, M.; Ertl, T. S.; Eberhardinger, U.; Bertagnolli, H.; SchmittWillich, H.; Albert, K. J. Phys. Chem. B 2003, 10\%, 4879-4886.

(13) Ernst, R. R.; Anderson, W. A. Rev. Sci. Instrum. 1966, 37, 93-102.

(14) Takahashi, M.; Chiba, K.; Li, P. J. Phys. Chem. B 2007, 111, 1343-1347. 
(15) Bruck, D.; Dudley, R.; Fyfe, C.; Delden, J. V. J. Magn. Reson. 1981, 42, 51-59.

(16) Zhang, Y.; Laude, D. A. J. Magn. Reson. 1990, 87, 46-55.

(17) Fischer, H.; Tseng, L.-H.; Raitza, M.; Albert, K. Magn. Reson. Chem. 2000, 38, 336342.

(18) Fyfe, C. A.; Cocivera, M.; Damji, S. W. H. Acc. Chem. Res. 1978, 11, 277-282.

(19) Chen, C.-H.; Shih, W.-C.; Hilty, C. J. Am. Chem. Soc. 2015, 137, 6965-6971.

(20) Ciobanu, L.; Jayawickrama, D. A.; Zhang, X.; Webb, A. G.; Sweedler, J. V. Angew. Chem. Int. Ed. 2003, 42, 4669-4672.

(21) Schroeder, F. C.; Gronquist, M. Angew. Chem. Int. Ed. 2006, 45, 7122-7131.

(22) Kc, R.; Gowda, Y. N.; Djukovic, D.; Henry, I. D.; Park, G. H.; Raftery, D. J. Magn. Reson. 2010, 205, $63-68$.

(23) Zimmerman, W. B.; Hewakandamby, B. N.; Tesař, V.; Hemaka Bandulasena, H.; Omotowa, O. A. Food Bioprod. Process. 2009, 87, 215-227.

(24) Macnaughtan, M. A.; Hou, T.; Xu, J.; Raftery, D. Anal. Chem. 2003, 75, 5116-5123. 


\section{Graphical TOC Entry}

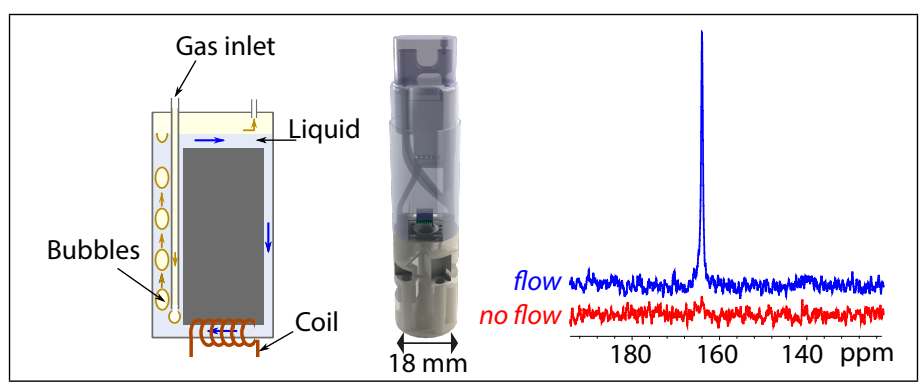

